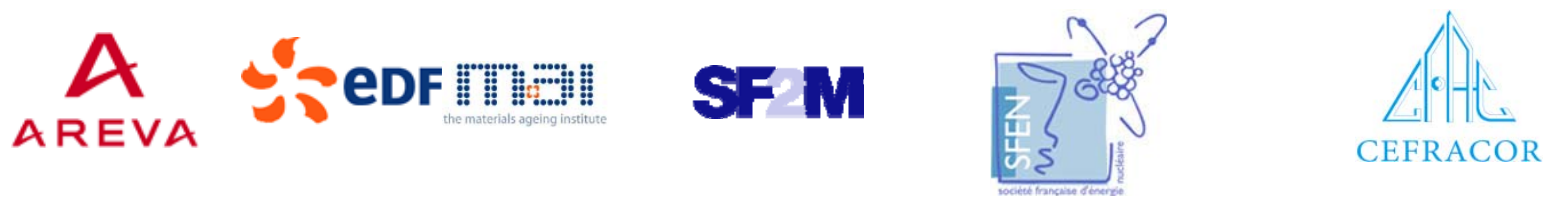

\section{MIN口S}

Centre of Excellence for Nuclear Materials

\section{Workshop}

Materials Innovation for Nuclear Optimized Systems
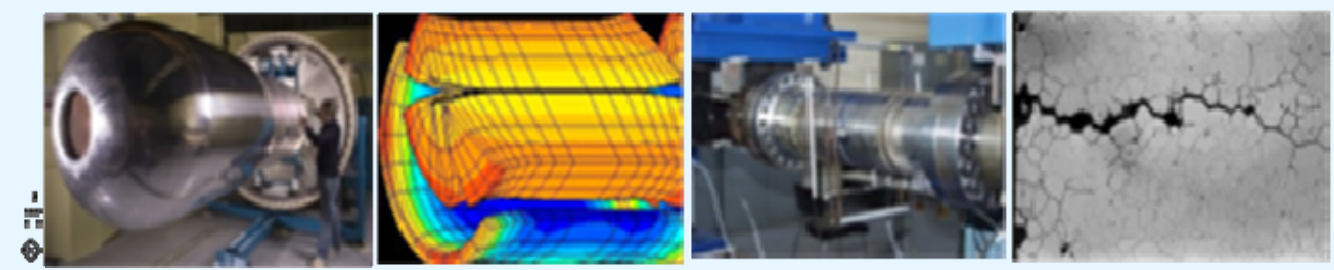

December 5-7, 2012, CEA - INSTN Saclay, France

\section{Lucile BECK}

CEA (France)

CEA Charged Particle Irradiation Facilities for Nuclear Material studies

Workshop organized by:

Christophe GALLÉ, CEA/MINOS, Saclay - christophe.galle@cea.fr Constantin MEIS, CEA/INSTN, Saclay - constantin.meis@cea.fr 
MINDS

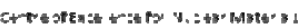

Workshop Materials Innovation for Nuclear Optimized Systems

December 5-7, 2012, CEA - INSTN Saclay, France

\title{
CEA Charged Particle Irradiation Facilities for Nuclear Material Studies
}

\author{
Lucile BECK ${ }^{1}$ \\ ${ }^{1}$ CEA-DEN-DMN, JANNUS Saclay laboratory (Saclay, FRANCE)
}

To study the effects of radiation on nuclear materials, the CEA uses various irradiation facilities producing neutrons or charges particles. Neutron facilities have the great advantage to directly produce neutron damages, but have also some drawbacks. The samples are activated and consequently their characterizations need dedicated hot cells, the reactors have fixed experimental parameters and the experiments are long and costly.

In order to better understand the mechanisms of radiation damage, experimental simulation can be also conducted with charged particles. In this case, experimental irradiation conditions (temperature, dose, flux, energy) are well controlled and the irradiated samples can be characterized with conventional analytical methods. This presentation will describe the CEA facilities devoted to ion or electron irradiations for material studies. Examples of irradiations performed at JANNUS (Joint Accelerators for Nano-science and NUclear Simulation) will be detailed (Fig. 1).

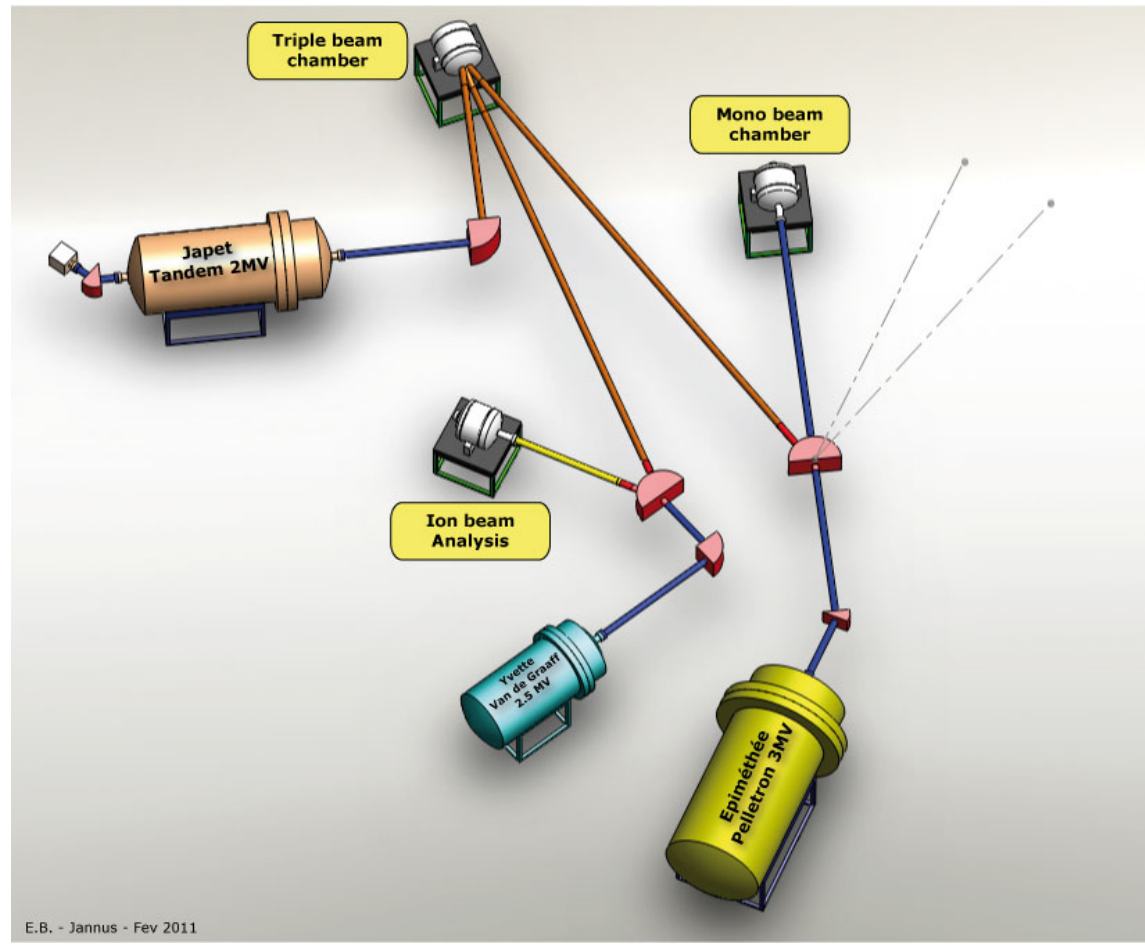

Fig. 1: Schematic view of the JANNUS platform at Saclay. 
DE LA RECHERCHE À L'INDUSTRIE
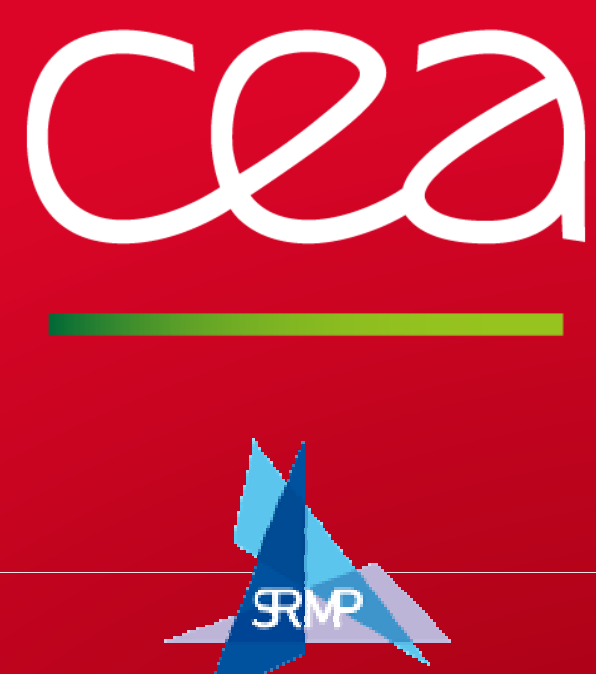

CEA charged particle irradiation facilities for nuclear material studies

\section{Lucile Beck}

CEA/DMN/SRMP/Jannus Laboratory - Saclay with the contributions of

B. Boizot (LSI), S. Bouffard \& M. Toulemonde (CIMAP) J. Henry \& A. Jankowiak (SRMA)

P. Trocellier, S. Pellegrino, S. Miro, Y. Serruys (JANNUS)

X. Deschanels (ICSM)

MINOS Workshop, Materials Innovation for Nuclear Optimized Systems December 5-7, 2012, CEA - INSTN Saclay, France 


\section{Cea PART 1 : CEA charged particle irradiation facilities}

MINDS

\section{lons}

- CIMAP - Caen (CEA/DSM, CNRS/INP, ENSICAEN, Caen University)

- JANNUS - Saclay (CEA/DEN/DMN/SRMP)

[GIS with JANNUS - Orsay (CNRS, Orsay University)]

\section{Electrons}

- LSI - Palaiseau (CEA/DSM, CNRS/INP, Ecole Polytechnique)

- THT - Saclay (CEA/DEN/DMN/SRMA)

- Located near Paris and in Caen

- Two CEA divisions: Materials Sciences Division (DSM) Nuclear Energy Division (DEN)

- in collaboration with CNRS and Universities

within

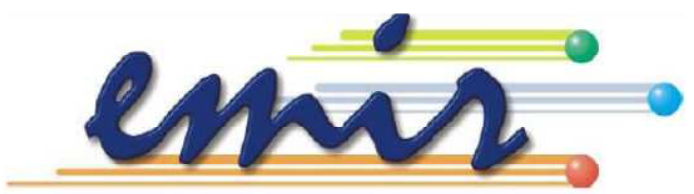




\section{EMIR network}

\section{a national network of accelerators dedicated to material irradiation}

EMIR network gathers the French facilities for material irradiation

- to promote the material research with the accelerators

- to meet for scientific challenge

- the prediction of the ageing of nuclear materials

- to achieve reliable simulations of the radiation damage through the study of model systems

- to develop irradiation as a tool for

- nano-structuration of materials

- solid state physics

- the physics of semiconductors

- to provide academic and industrial researchers access to irradiation facilities and online characterization

- to assess the need for development facilities

- to ensure the visibility of these facilities

A call for proposal of experiment every year : http://emir.in2p3.fr/EMIR-network Next call: October 2013 


\section{Large choice of particles and energies for different interactions}

MINDS

Scheme of energy deposition of an ion in matter / electrons in matter

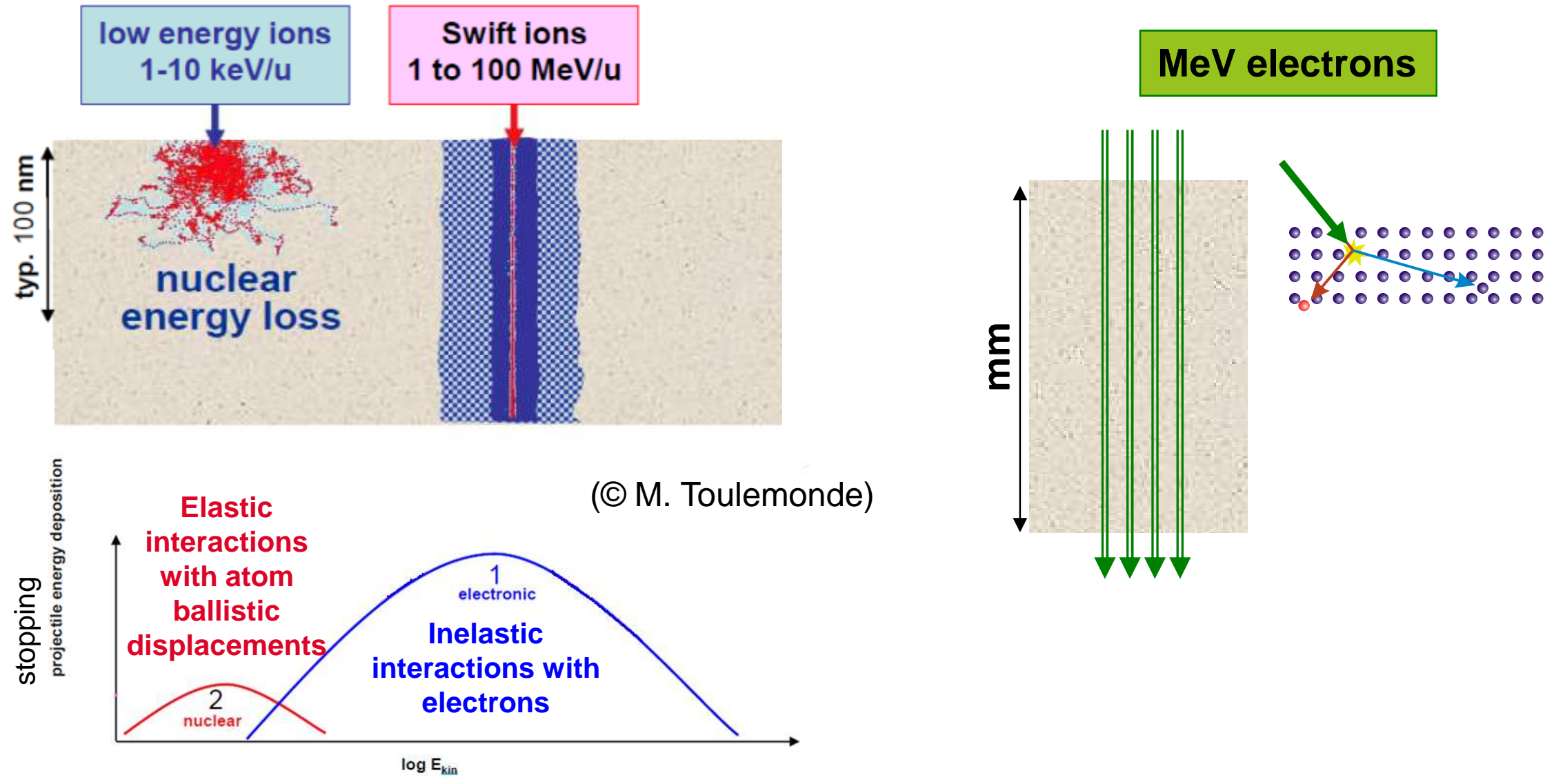




\section{Cea Access to the CEA charged particle irradiation facilities}

\section{MIN口S}

\section{emis: facilities}

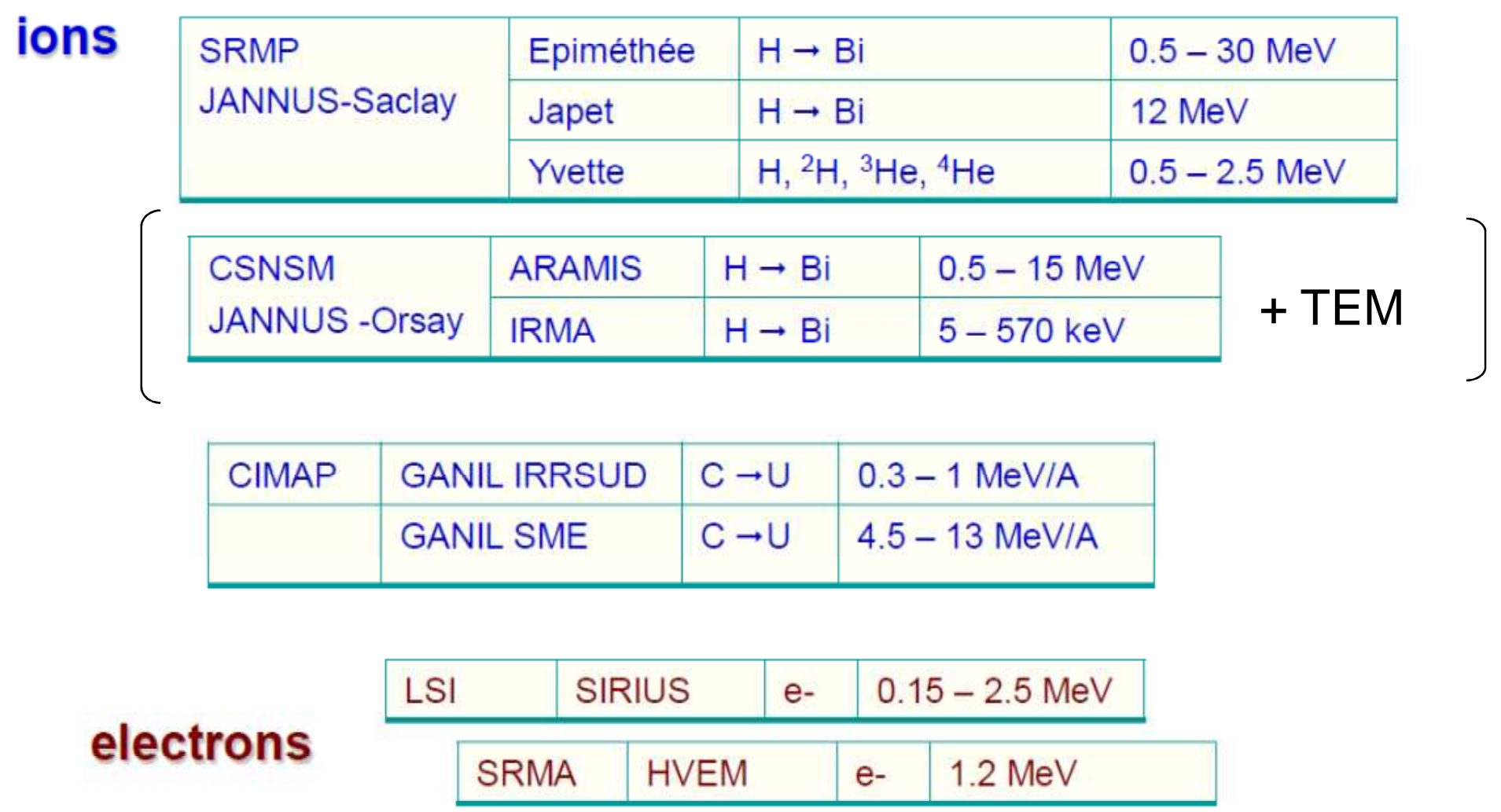




\section{Cea Cimap-Caen: medium to heavy ions - MeV energy range}

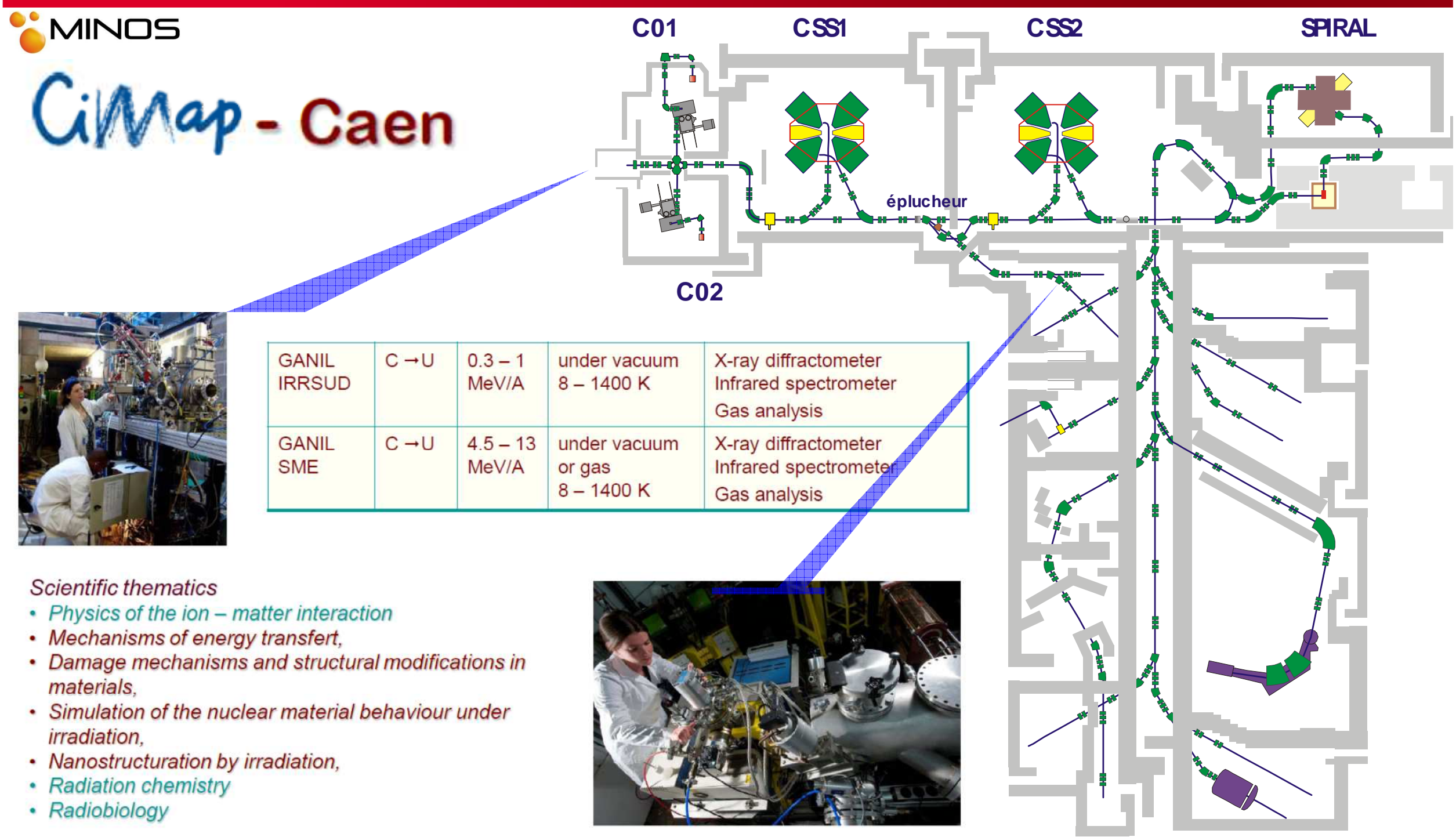




\section{JANNUS-Saclay: 3 joint accelerators}

\section{Low to heavy ions - MeV energy range}

\section{$\because$ MINDS}

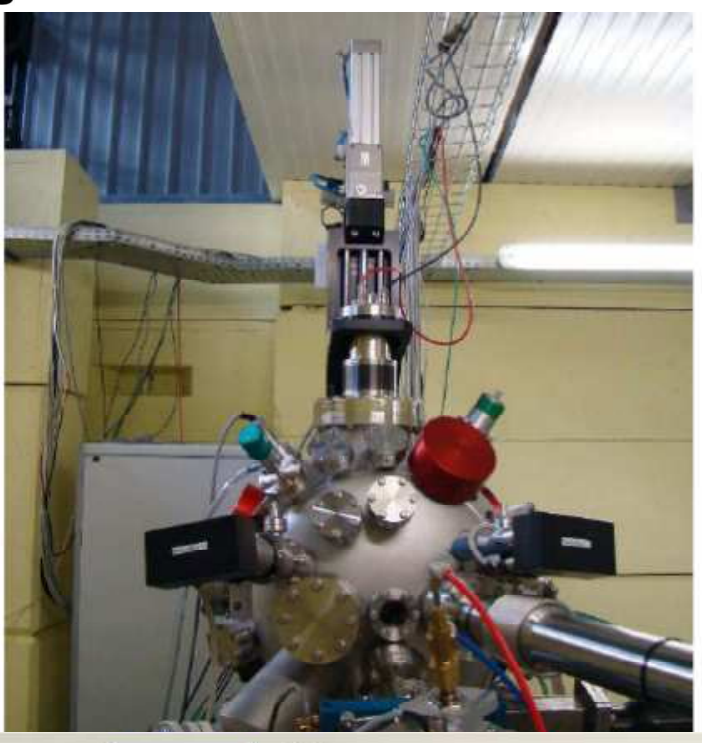

\begin{tabular}{|l|l|l|l|l|}
\hline Epiméthée & $\mathrm{H} \rightarrow \mathrm{Bi}$ & $\begin{array}{l}0.5-30 \\
\mathrm{MeV}\end{array}$ & $\begin{array}{l}\text { under vacuum } \\
77-800 \mathrm{~K}\end{array}$ & \\
\hline Japet & $\mathrm{H} \rightarrow \mathrm{Bi}$ & $\begin{array}{l}\text { tandem } \\
2 \mathrm{MV}\end{array}$ & $\begin{array}{l}\text { under vacuum } \\
77-800 \mathrm{~K}\end{array}$ & \\
\hline Yvette & $\begin{array}{l}\mathrm{H},{ }^{2} \mathrm{H}, \\
{ }^{3} \mathrm{He}, \\
{ }^{4} \mathrm{He}\end{array}$ & $\begin{array}{l}0.5- \\
2.5 \mathrm{MeV}\end{array}$ & under vacuum & $\begin{array}{l}\text { NRA - RBS } \\
\text { ERDA - PIXE }\end{array}$ \\
\hline
\end{tabular}

Scientific thematics

- Microstructure evolution under irradiation for nuclear materials

- Synergetic effects of damage and Helium and Hydogen production

- Atomic transport phenomena under irradiation

- Controled modification by irradiation of the material properties

- Teaching and formation with the accelerators
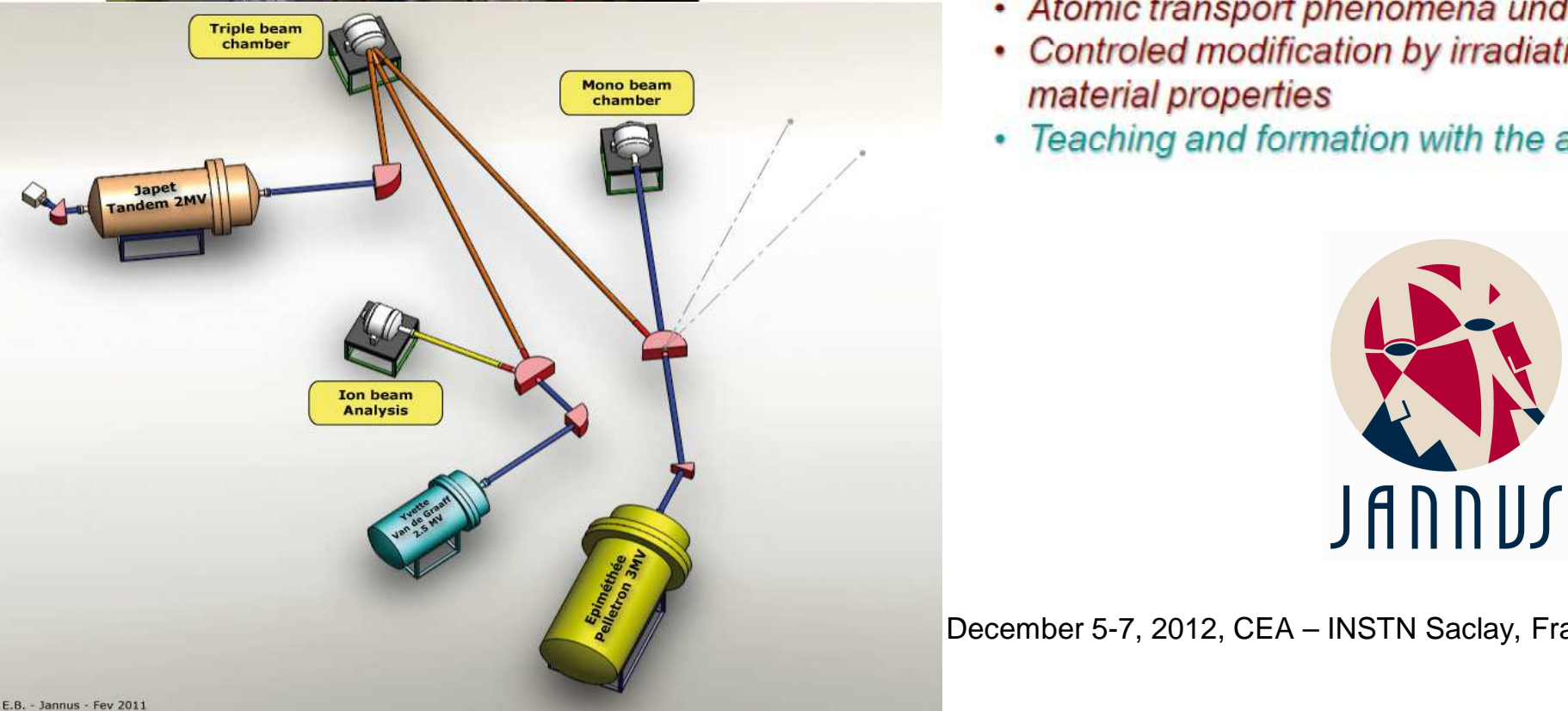


\section{Cea LSI-Palaiseau: MeV energy electrons}

\section{MINDS}

\section{LSI - Palaiseau}

\begin{tabular}{|l|l|l|l|l|l|}
\hline SIRIUS & e- & $\begin{array}{l}0.15-2.5 \\
\mathrm{MeV}\end{array}$ & $\begin{array}{l}\text { High doses } \\
\text { (GGy) } \\
\text { Large area } \\
20-600 \mathrm{~K}\end{array}$ & $\begin{array}{l}\text { Optical spectroscopies } \\
\text { Micro-electrochemistry } \\
\text { Electrical measurements } \\
\text { Gas emission }\end{array}$ \\
\hline
\end{tabular}

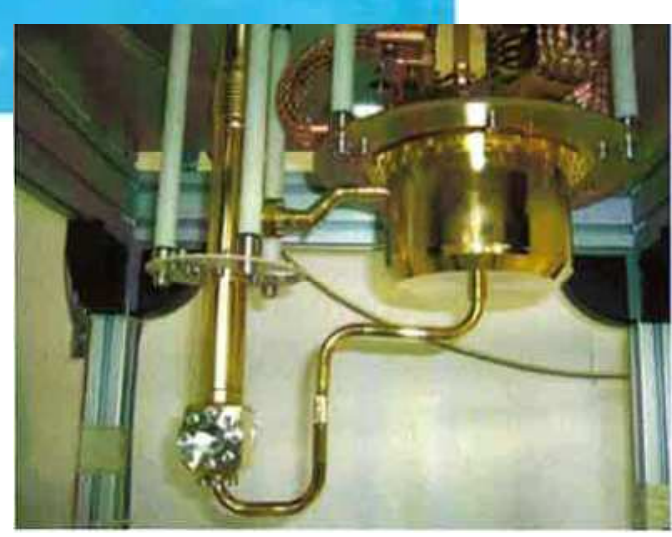

Scientific thematics

- Irradiation effects on material properties

- Material ageing under irradiation

- Characterization of induced defects (defect type, displacement threshold)

- Radio-grafting of polymers

- Corrosion under irradiation 


\section{Cea SRMA-Saclay: MeV energy electrons}

$\because \mathrm{MINC}$

$$
\text { SRMA - Saclay }
$$

\begin{tabular}{|l|l|l|l|l|}
\hline HVEM & e- & $0.3-1.2 \mathrm{MeV}$ & under vacuum & TEM \\
\hline
\end{tabular}

Scientific thematics

- Microstructure evolution under irradiation for nuclear materials

- Effects of the high damage rate
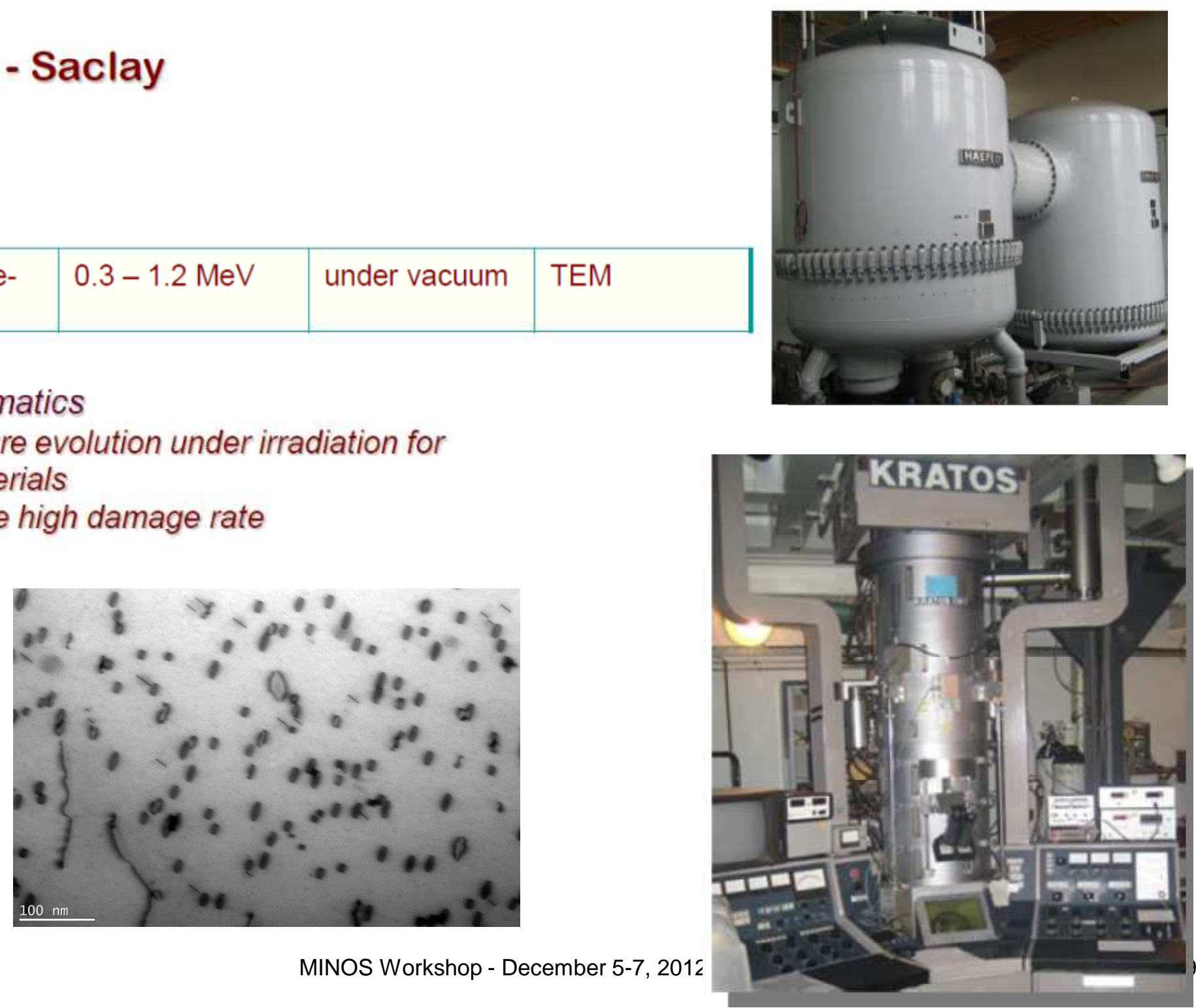


\section{Cea PART 2: examples}

\section{$\because$ MINDS}
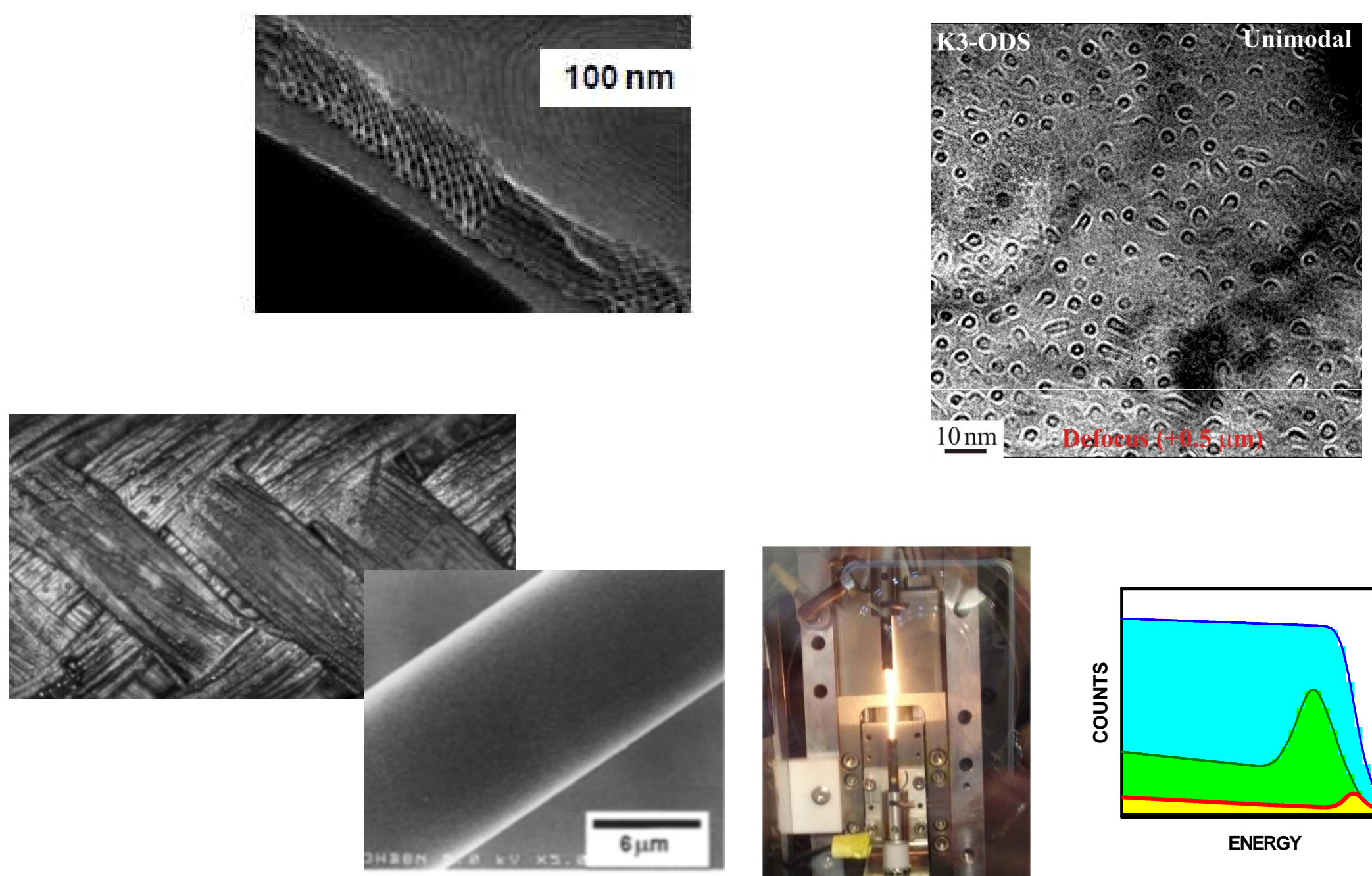


\section{Example 1: Mesoporous silica gels for applications in the field of nuclear waste}

MINDS

\begin{tabular}{l}
-Sample characteristics \\
-Si substrate \\
-Some samples filled with $\mathrm{C}$ and Cs to simulate a radionuclide \\
-Layer thickness : 100nm \\
\hline SBA15: Structure 2D hexagonale
\end{tabular}

Separation chemistry

Waste management

$X$. Deschanels, S. Dourdain and coll. ICSM, Marcoule 


\section{Cea Example 1: Mesoporous $\mathrm{SiO}_{2}$ gel}

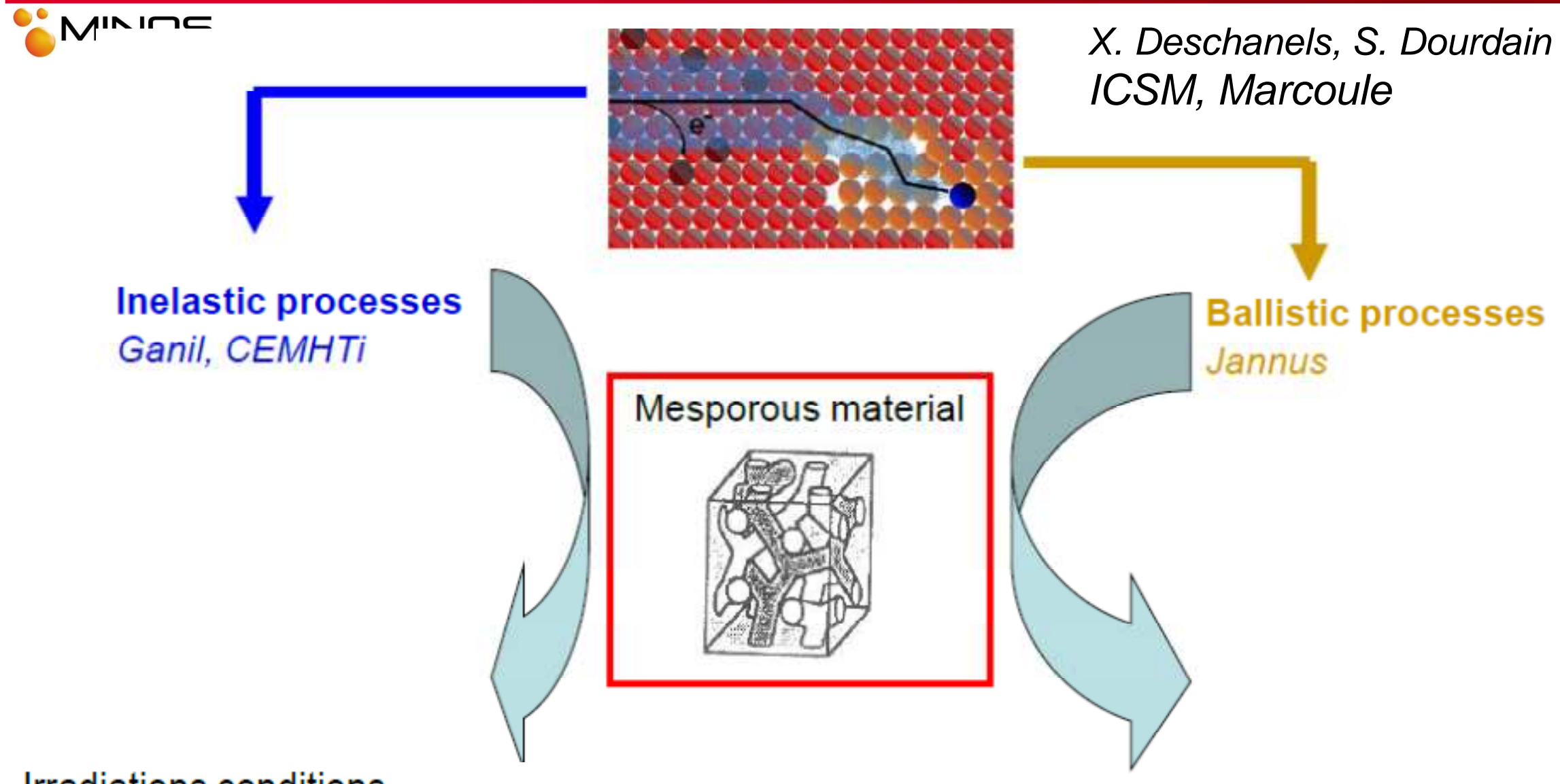

Irradiations conditions

Inelastic processes $\mathrm{Xe}: 92 \mathrm{MeV}$
$-1 \times 10^{13} \mathrm{~cm}^{-2}-\mathrm{T}_{\text {amb. }}$
$(\mathrm{dE} / \mathrm{dx})_{\mathrm{e}}=9 \mathrm{keV} / \mathrm{nm}$

$20 \mathrm{MeV} \operatorname{Ar}\left(\mathrm{S}_{\mathrm{e}}=3,4 \mathrm{keV} / \mathrm{nm}\right)$

$0,5 \mathrm{MeV} \mathrm{He}\left(S_{e}=0,26 \mathrm{keV} / \mathrm{nm}\right) @ R T$ 


\section{Example 1: Mesoporous $\mathrm{SiO}_{2}$ gel}

MINDS
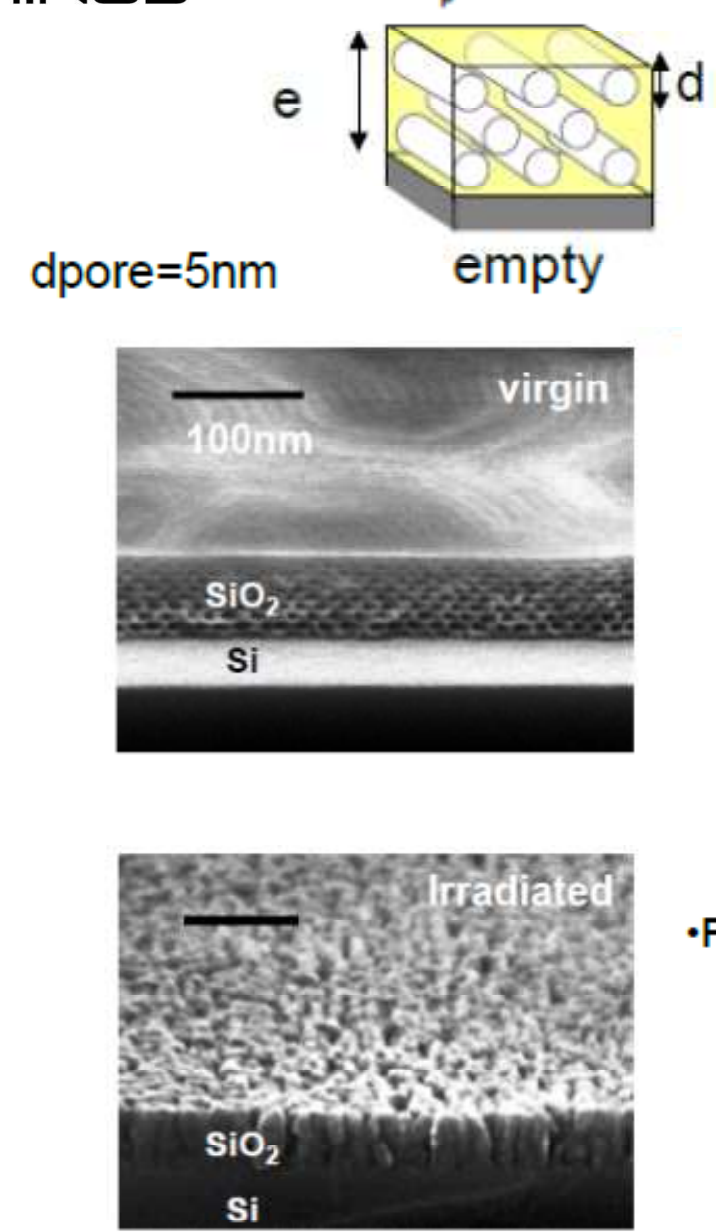

-Parameters of the coating

$\checkmark$ Thickness decrease

$\checkmark$ Porosity volume increase

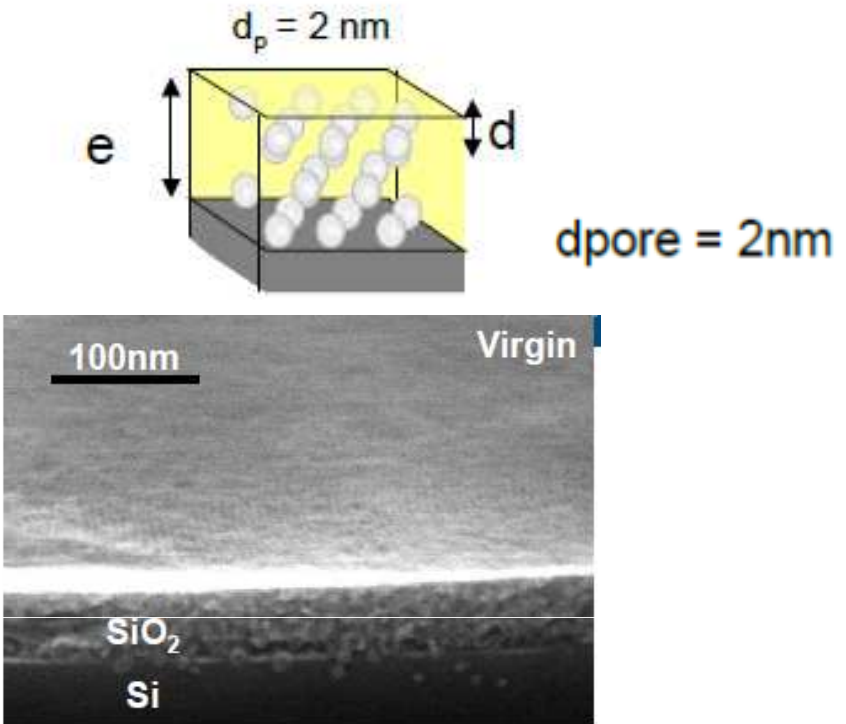

Irradiated

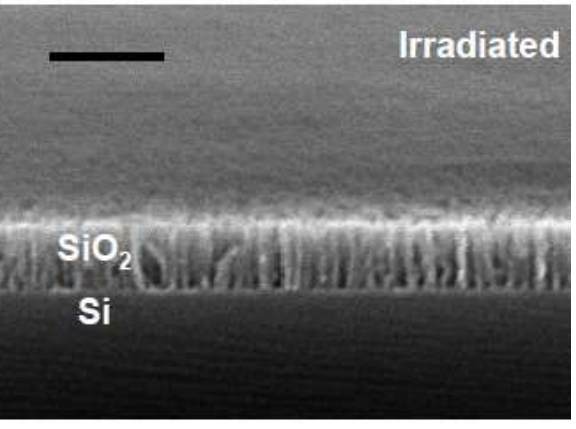

- Destructuration of the silica network under Xe 92MeV 


\section{Cea Example 1: Mesoporous $\mathrm{SiO}_{2}$ gel}

MINDS

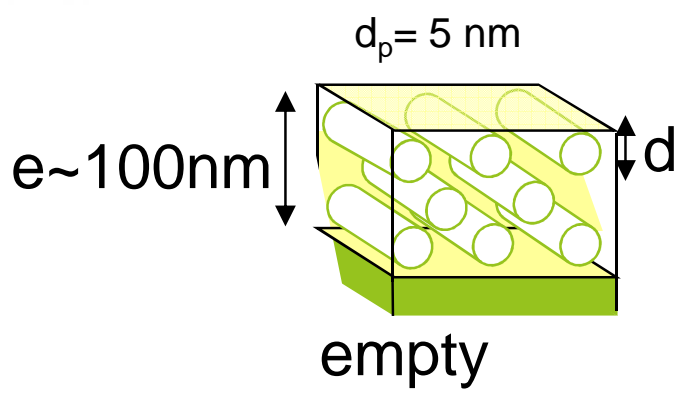

\section{$0.5 \mathrm{MeV} \mathrm{He}, 20 \mathrm{MeV}$ Ar @ JANNUS}
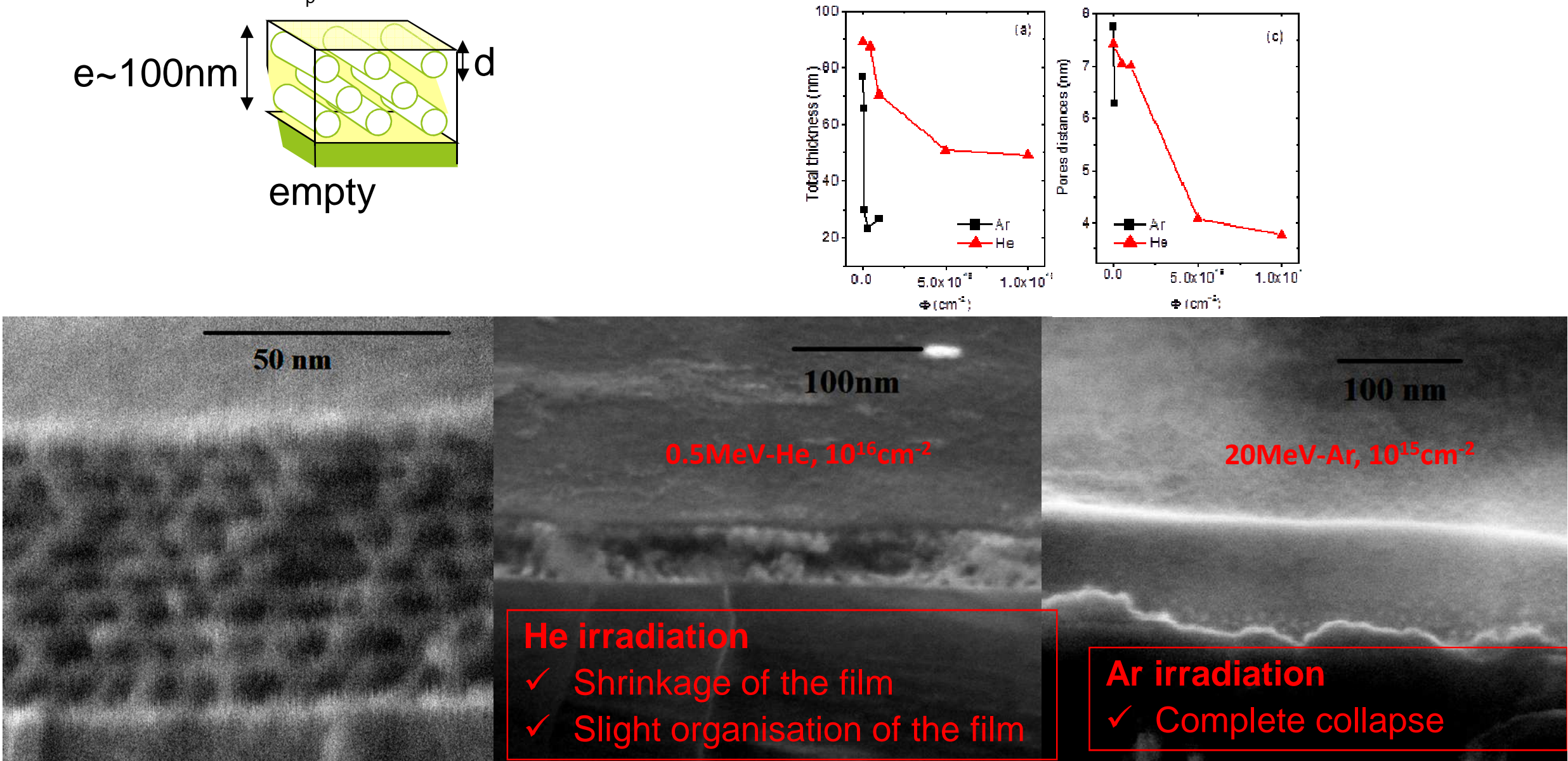

Densification and loss in the organisation of the thin films even for $S_{e}$ lower than track threshold 


\section{Cea Example 2: In-situ mechanical test}

¿MINDS

$\underline{\text { IRRSUD line GANIL Caen }}$

Épiméthée E3 line JANNUS Saclay

$\Rightarrow$ Damage effect for both electronic and nuclear slowing down regimes
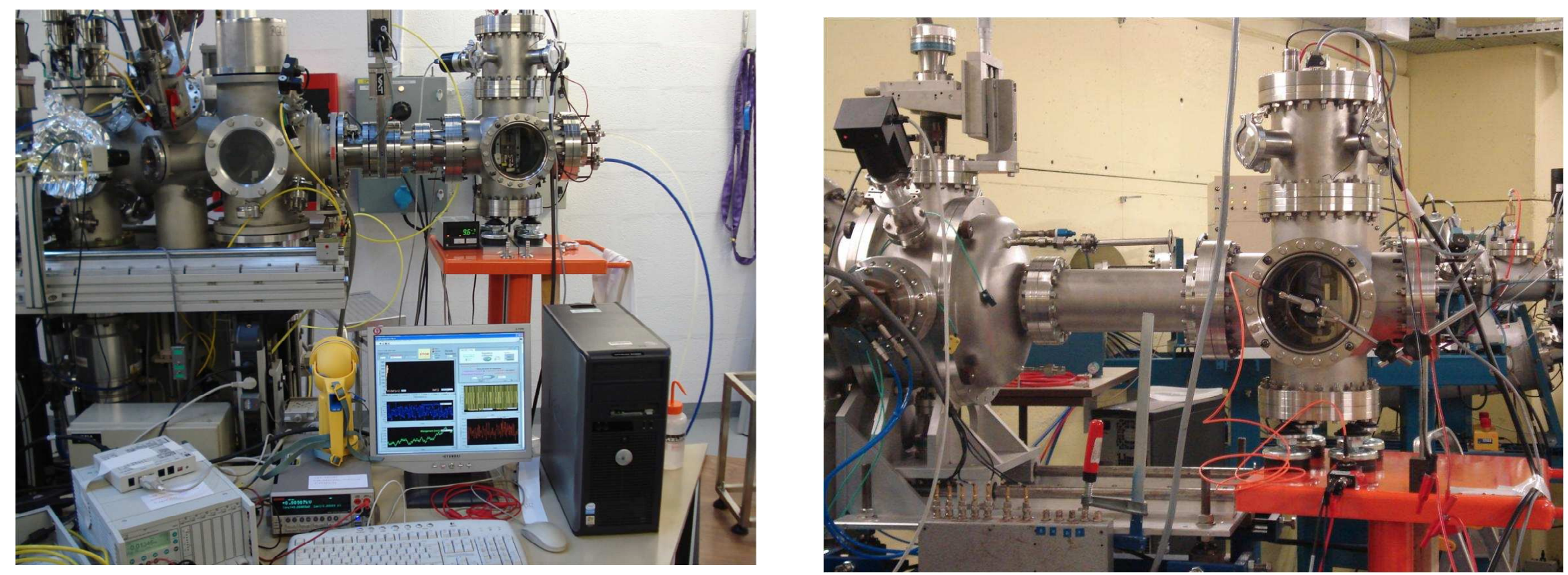

A. Jankowiak, Ch. Colin (CEA/DM//SRMA) et coll. 


\section{Example 2: In-situ mechanical test}

\section{MIN口S}

\section{Development of a in-situ tensile test device}
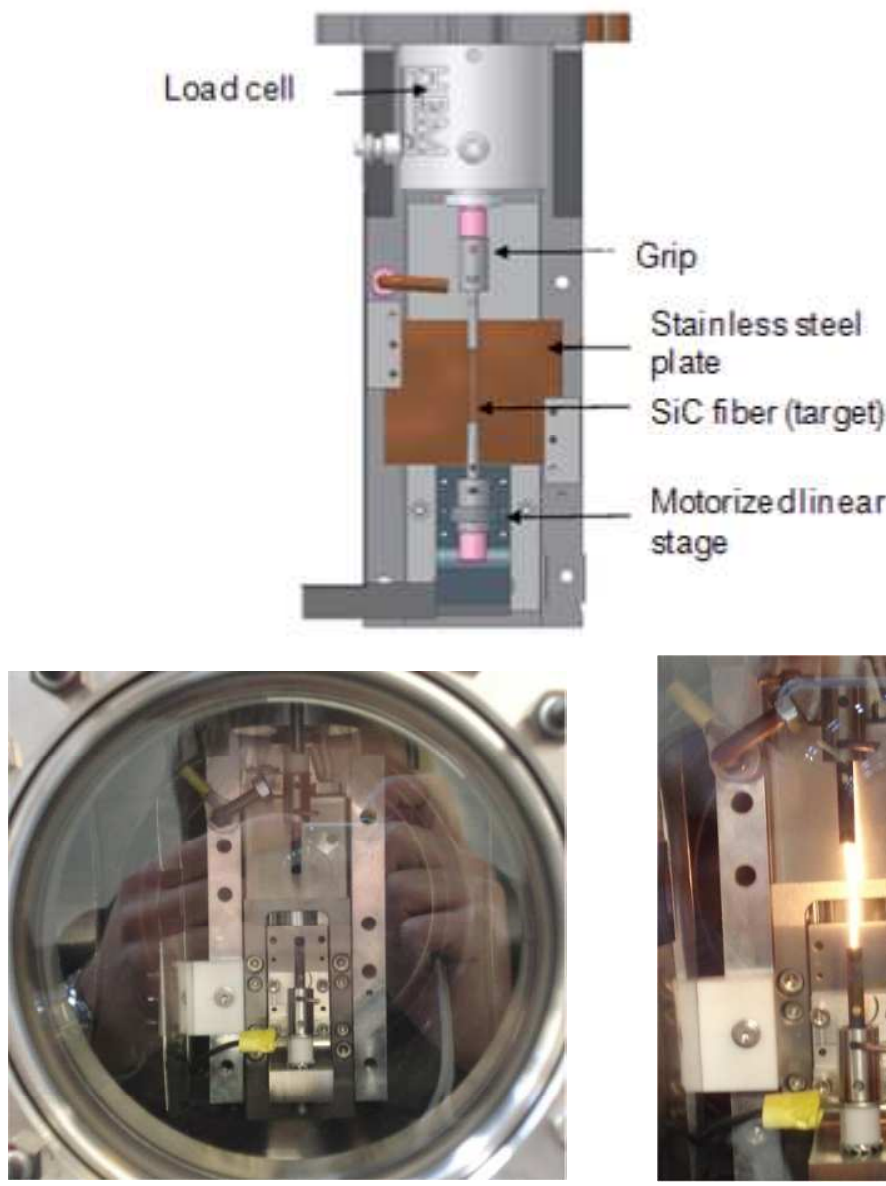

CEA - DEN

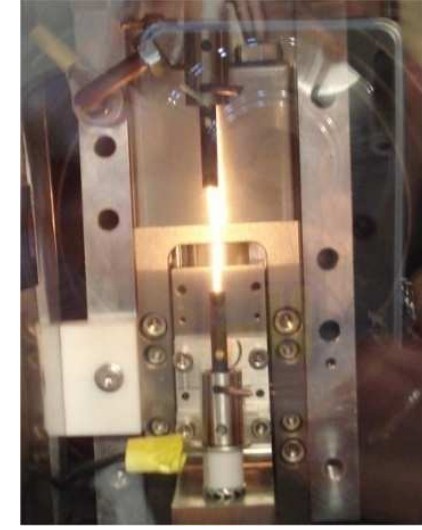

MINOS Workshop - December 5-7, 2012, CEA - INSTN Saclay, France $\checkmark$ Tensile test on single $\mathrm{SiC}$ fiber up to $5 \mathrm{~N}$;

$\checkmark$ Usable for other type of materials;

$\checkmark$ Gauge length: $25 \mathrm{~mm}$;

$\checkmark$ In-situ measurements of the fiber diameter possible;

$\checkmark$ Operated in vacuum: $10^{-6}$ to $10^{-7} \mathrm{mbar}$;

$\checkmark$ Tensile tests (creep tests) from $25^{\circ} \mathrm{C}$ à $1800^{\circ} \mathrm{C}$;

$\checkmark$ Graphite-grip method: cold grips and uniform temperature $\checkmark$ Implementable on every standard type of beam line; 


\section{Example 2: In-situ mechanical test}

\section{MIN口S}

\section{In situ tensile tests on SiC fibers under ion beam}

IRRSUD line GANIL Caen $\mathrm{Xe}^{23+} 92 \mathrm{MeV}$

Épiméthée E3 line JANNUS Saclay C+ 9 and 12 MeV

Fiber at $1000{ }^{\circ} \mathrm{C}$ and $300 \mathrm{MPa}$
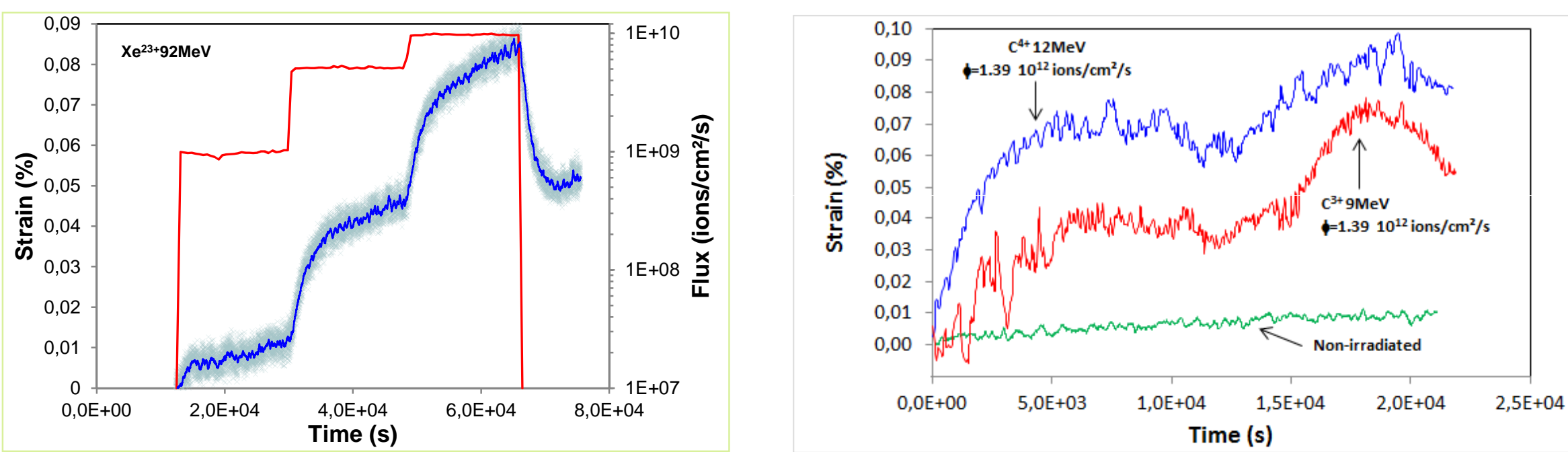

Fiber at $1000{ }^{\circ} \mathrm{C}$ and $300 \mathrm{MPa}$ (irradiation-enhanced $\mathrm{c}$ reep study)

- Strain rate variation with flux intensity;

- Residual stain at $1000{ }^{\circ} \mathrm{C}<0.1 \%$ for $2.10^{12} 92 \mathrm{MeV} \mathrm{Xe} e^{23+}$ ions $/ \mathrm{cm}^{2}$ and $3.10^{16} 12 \mathrm{MeV} \mathrm{C}^{4+}$ ions $/ \mathrm{cm}^{2}$

Previous works carried out at RT (irradiation-induced swelling study)

Residual strain at RT 0,50\% \% for $5.10^{14} 92 \mathrm{MeV} \mathrm{Xe} 23+$ ions $/ \mathrm{cm}^{2}$ and $~ 0.45 \%$ for $10^{17} 12 \mathrm{MeV} \mathrm{C}^{4+}$ ions $/ \mathrm{cm}^{2}$ Annealing test at $1800^{\circ} \mathrm{C}$ : residual strain almost disapp ears completely 


\section{Example 3: STUDY OF RADIATION EFFECTS IN CARBIDES (SIC, TiC, ZrC)}

MIN口S

$\mathrm{PhD}$ thesis of $\mathrm{S}$. Pellegrino (JANNUS): "Irradiation effects in carbides (SiC, TiC and $\mathrm{ZrC}$ ) and synergetic effects of electronic and nuclear energy losses."

\section{GANIL-SME}

Pb 900 MeV

High Energy

$(\mathrm{dE} / \mathrm{dx})_{\text {nucl. }}<<(\mathrm{dE} / \mathrm{dx})_{\text {elec. }}$

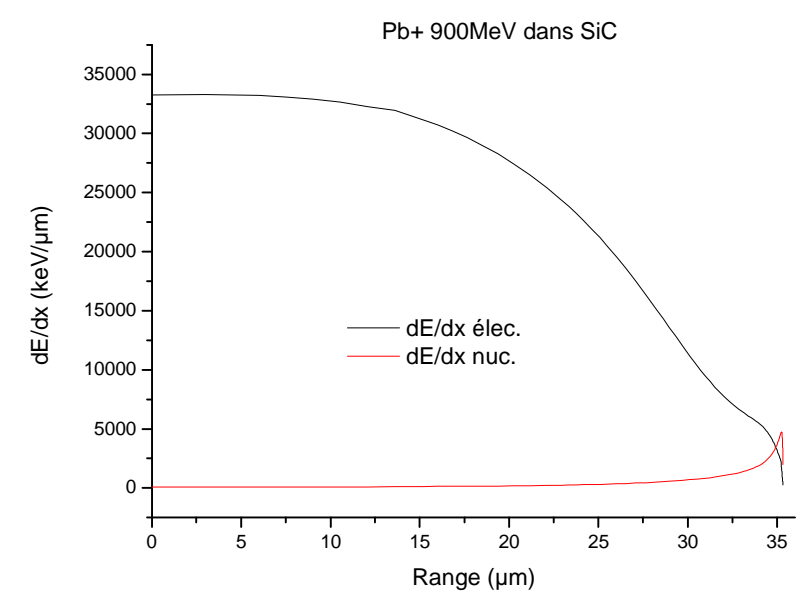

JANNUS

Au 1,2 MeV

Low Energy

$(d E / d x)_{\text {nucl. }}>(d E / d x)_{\text {elec. }}$

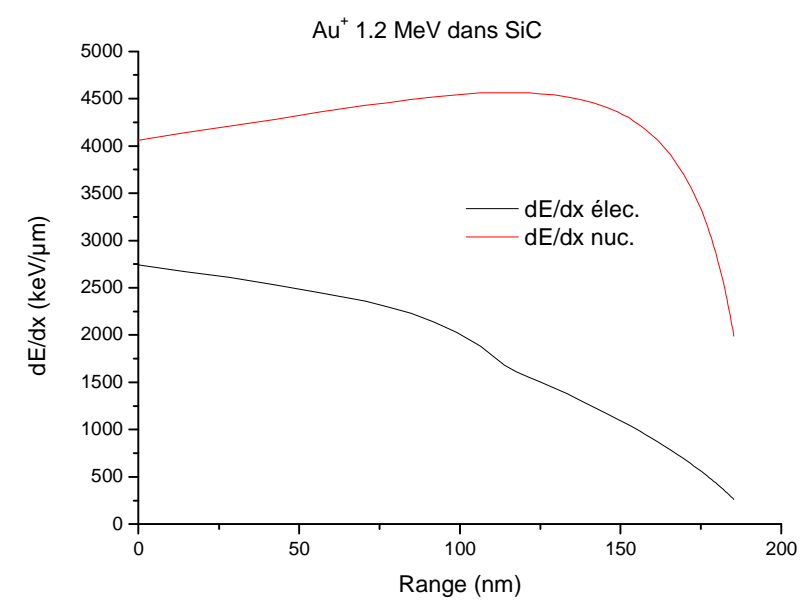




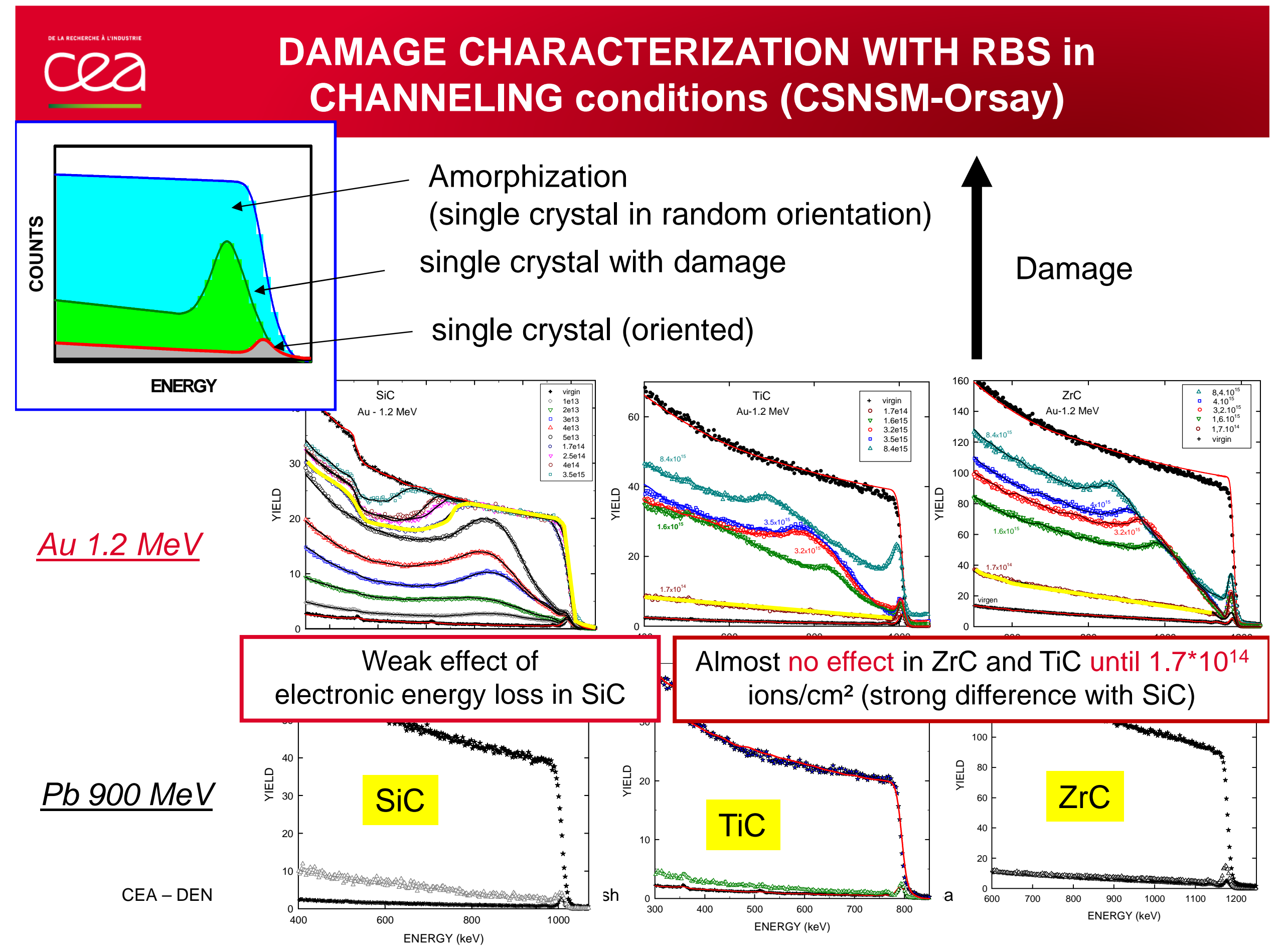




\section{Cea EXAMPLE 4: ODS (OXIDE DISPERSED STRENGTHENED) STEELS}

\section{$\because$ MINDS}

\begin{tabular}{|c|}
\hline $\begin{array}{c}\text { Dual beam } \\
\text { irradiation }\end{array}$ \\
\hline
\end{tabular}

M. J. Fluss, S. Tumey, L. Hsiung (LLNL) and A. Kimura (Kyoto University)

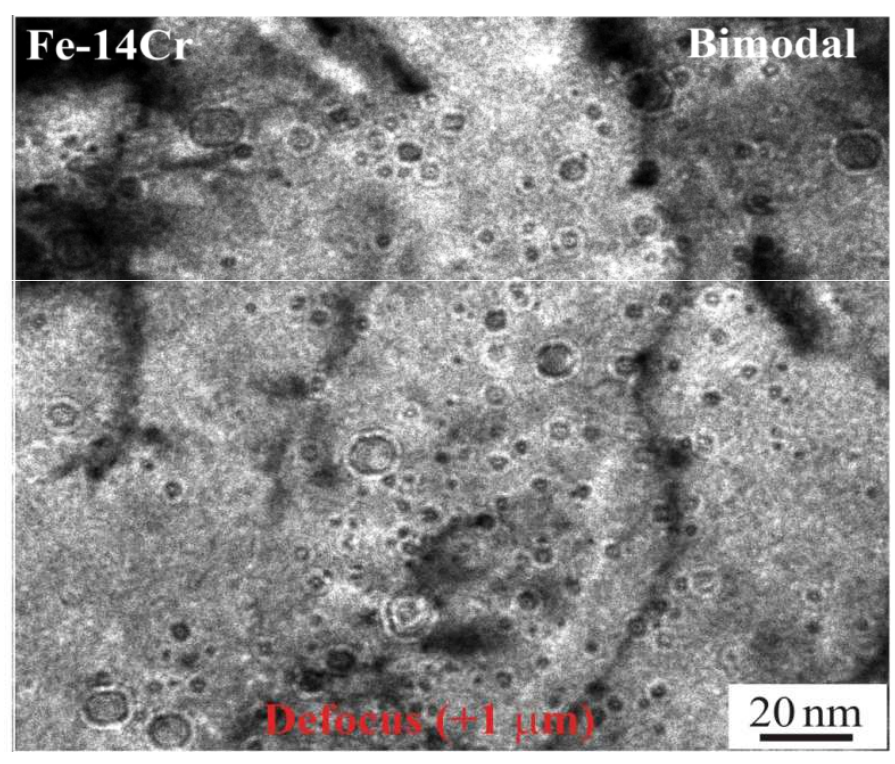

Start of void growth. Swelling

\section{ODS (K3 and MA957) Fe-Cr 14\%}
${ }^{56} \mathrm{Fe}^{8+} \quad 24 \mathrm{MeV} \quad \underline{{ }^{4} \mathrm{He}^{+}} 1.7 \mathrm{MeV}$ (degraded with carbon foils)
Dose $\sim 30 \mathrm{dpa} \quad[\mathrm{He}] \sim 25 \mathrm{appm} / \mathrm{dpa}$

A comparison of a steel with nano-particles (right) and a steel without the nano-particles (left). The specimen with particles exhibits small bubbles all below the critical size.

The specimen without particles shows the start of void growth.

L. Hsiung, M. Fluss et al. (LLNL \& SRMP), Phys. Rev. B 82, 2010, 184103 


\section{Cea EXAMPLE 4: ODS (OXIDE DISPERSED STRENGTHENED) STEELS}

\section{J. Fluss, S. Tumey, L. Hsiung (LLNL) and A. Kimura (Kyoto University)}

\section{MIN口S}

\section{Dual beam irradiation, details}
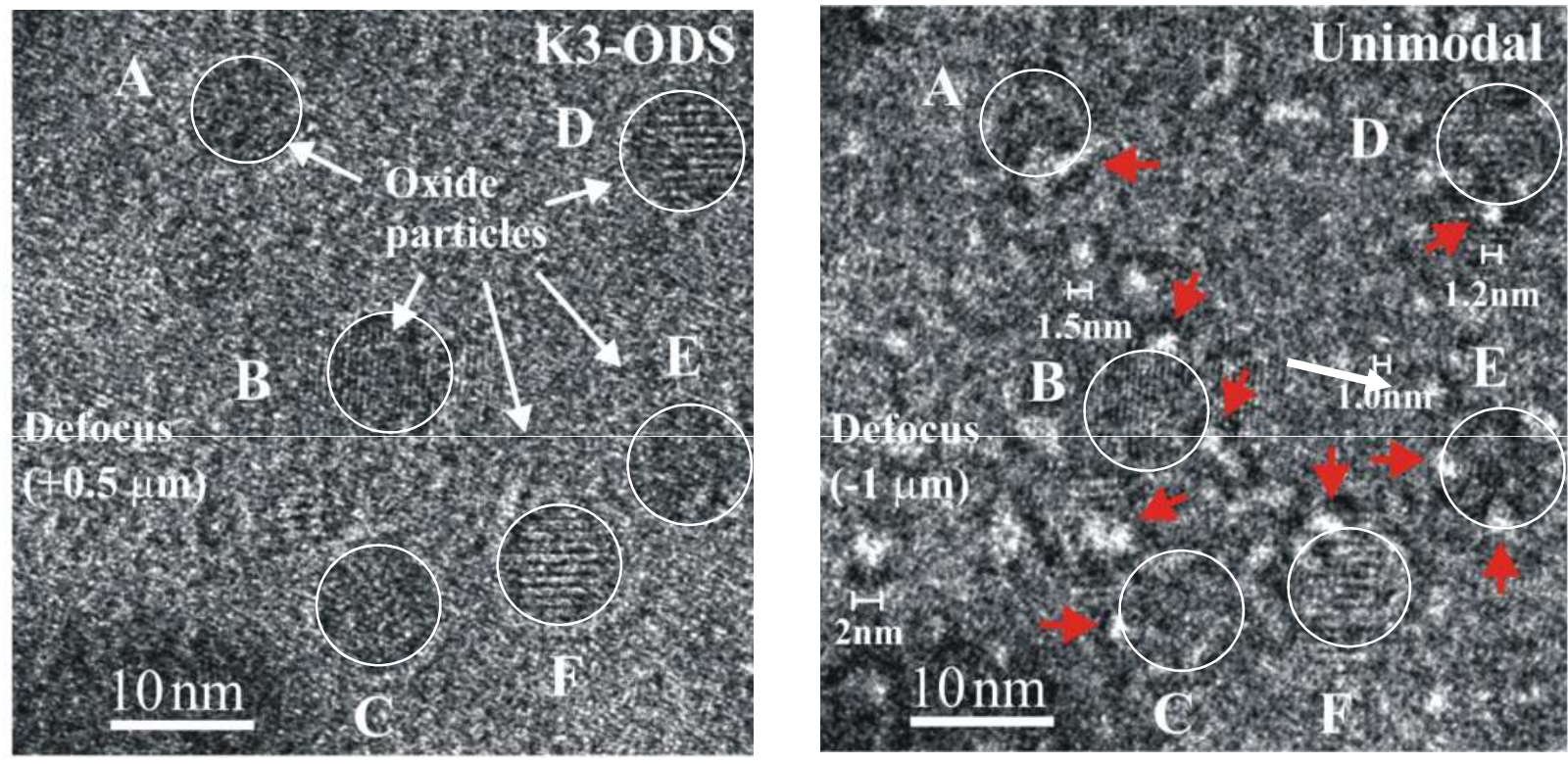

Oxides nano-particles (labeled A thru F)

By varying the focusing condition on the right we see the small He bubbles (red arrows) trapped at the interface between the particles and the steel matrix.

Oxide nanoparticles play the role of defect sinks by trapping $\mathrm{He}$ and preventing swelling. 


\section{Ceح EXAMPLE 4: ODS (OXIDE DISPERSED STRENGTHENED)STEELS}

\section{MINDS}

Triple beam irradiation

M. J. Fluss, S. Tumey, L. Hsiung (LLNL) and A. Kimura (Kyoto University)

\section{ODS (K3 and MA957), $\mathrm{Fe}$ and $\mathrm{Fe}-\mathrm{Cr}$ 14\%}

\begin{tabular}{c|c|c}
$\frac{56 \mathrm{Fe}^{8+}}{24 \mathrm{MeV}}$ & $\begin{array}{c}\frac{4 \mathrm{He}^{+}}{7.7 \mathrm{MeV}} \\
\text { (degraded with carbon } \\
\text { foils) }\end{array}$ & $\begin{array}{c}\underline{\mathrm{H}^{+}} \\
52 \mathrm{keV} \\
\begin{array}{c}\text { (degraded with carbon } \\
\text { foils) }\end{array} \\
\text { Dose } \sim 30\end{array}$ \\
{$[\mathrm{He}] \sim 25 \mathrm{appm} / \mathrm{dpa}$} & {$[\mathrm{H}] \sim 70$ appm/dpa } \\
$\mathrm{T}=425 \mathscr{C}$ &
\end{tabular}
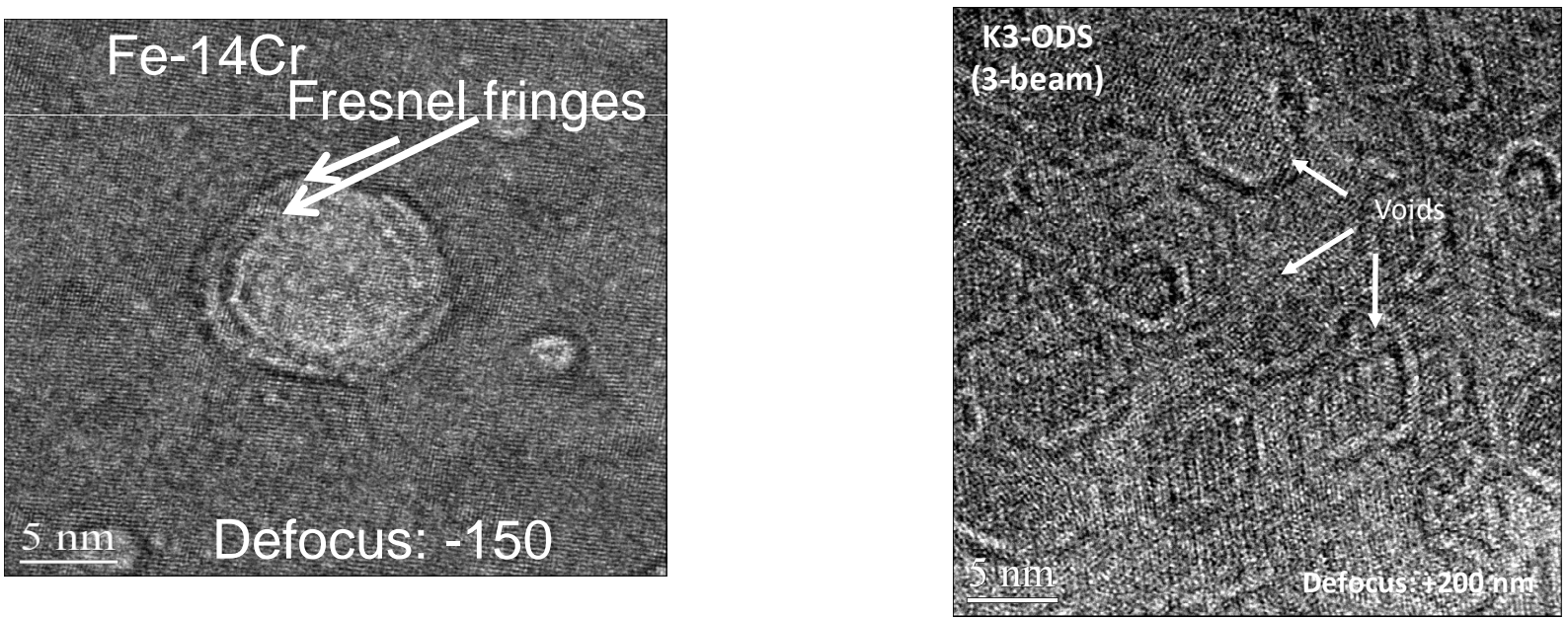

The cavities exhibit a double shell structure 


\section{Summary}

Electron and ion irradiation experiments

\section{Electron: $0.15-2.5 \mathrm{MeV}$}

>lons: $\mathrm{H}$ to $\mathrm{U}, 0.3-1000 \mathrm{MeV}$

Combined with in situ or ex situ instrumentations:

- Low to high temperature: $8 \mathrm{~K}-1200 \mathrm{~K}$

- X-ray diffractometer

- IR spectrometer

- Gas analyser

- IBA

- UV-visible

- Electrical measurements

- Raman spectrometer

- TEM

Next call: October 2013 for the period of April 2014 to March 2015 http://emir.in2p3.fr/EMIR-network Next EMIR User days in 2013

Visit of JANNUS-Saclay on Friday 


\section{MIN口S}

\begin{tabular}{|c|c|c|c|c|c|c|}
\hline Samples & $\begin{array}{c}\text { Single crystal } \\
6 \mathrm{H}-\mathrm{SiC}\end{array}$ & $\begin{array}{c}\text { Single crystal } \\
\text { TiC }\end{array}$ & $\begin{array}{c}\text { Single crystal } \\
\text { ZrC }\end{array}$ & $\begin{array}{c}\text { Single crystal } \\
6 \mathrm{H}-\mathrm{SiC}\end{array}$ & $\begin{array}{c}\text { Single crystal } \\
\text { TiC }\end{array}$ & $\begin{array}{c}\text { Single crystal } \\
\text { ZrC }\end{array}$ \\
\hline Facility & JANNUS & JANNUS & JANNUS & GANIL-SME & GANIL-SME & GANIL-SME \\
\hline Ion & $\mathrm{Au}^{+}$ & $\mathrm{Au}^{+}$ & $\mathrm{Au}^{+}$ & $\mathrm{Pb}^{53+}$ & $\mathrm{Pb}^{53+}$ & $\mathrm{Pb}^{53+}$ \\
\hline Energy $(\mathrm{MeV})$ & 1.2 & 1.2 & 1.2 & 956 & 956 & 956 \\
\hline Projected Range: $\mathrm{R}_{\mathrm{p}}$ & $194 \mathrm{~nm}$ & $219 \mathrm{~nm}$ & $217 \mathrm{~nm}$ & $37.13 \mu \mathrm{m}$ & $28.2 \mu \mathrm{m}$ & $26.5 \mu \mathrm{m}$ \\
\hline $\mathrm{dpa}$ & $0.05-40.25$ & $0.066-55.4$ & $0.1-83.5$ & $0.0002-0.0013$ & $0.0002-0.0012$ & $0.0003-0.0025$ \\
\hline$(\mathrm{dE} / \mathrm{dx})_{\mathrm{e}}(\mathrm{keV} / \mu \mathrm{m})$ & 2728 & 2147 & 1689 & 33080 & 43580 & 47160 \\
\hline$(\mathrm{dE} / \mathrm{dx})_{\mathrm{n}}(\mathrm{keV} / \mu \mathrm{m})$ & 4051 & 3636 & 3736 & 61.03 & 81.62 & 102 \\
\hline Fluences$\left(\mathrm{ions} / \mathrm{cm}^{2}\right)$ & $10^{13}-10^{16}$ & $10^{13}-10^{16}$ & $10^{13}-10^{16}$ & $10^{12}-10^{13}$ & $10^{12}-10^{13}$ & $10^{12}-10^{13}$ \\
\hline
\end{tabular}

